\title{
Overestimating the effects of healthy aging
}

\author{
Vanja Kljajevic * \\ University of the Basque Country (UPV/EHU), Vitoria \& IKERBASQUE, Basque Foundation for Science, Bilbao, Spain
}

Keywords: cognitive aging, Alzheimer's disease, biomarkers, amyloidosis, neurodegeneration, neuroimaging

Typical cognitive aging is often defined as aging free of dementia, and yet it does not seem to exclude dementia-related pathology. The most common type of dementia is Alzheimer's disease (AD) - it is a progressive neurodegenerative disease that affects multiple cognitive domains, including memory, attention, reasoning, judgment, language, as well as behavior. AD accounts for $60-80 \%$ of dementia cases, currently affecting 35 million people worldwide and progressing toward an estimated 115 million by the year 2050 (Riedel, 2014). Since there is no cure for AD, the research focus has shifted from the symptomatic stage to the earlier stages of the disease, with the goal to develop treatments that would effectively delay the onset of symptoms and progression of this devastating disease (Selkoe, 2012; Bateman, 2015). The need to diagnose and begin treatment even before overt symptoms appear has generated in neurological researchers a strong interest in cognitively healthy people who are at risk for $\mathrm{AD}$. Recent modifications of the research and diagnostic criteria for $\mathrm{AD}$ reflect this trend by including cognitively normal $(\mathrm{CN})$ at-risk-for-AD persons as a preclinical stage in the AD continuum (Dubois et al., 2007, 2010; Sperling et al., 2011).

Crucial in the modified criteria for AD is the concept of biomarkers of Alzheimer's pathology, i.e., physiological and anatomical parameters that can be objectively measured to establish the presence of changes due to the disease (Jack and Holtzman, 2013). Since amyloid- $\beta$ neuritic plaques and neurofibrillary tangles have been established as the pathological hallmarks of $\mathrm{AD}$, biomarkers of amyloidosis and neurodegeneration are used as in vivo indicators of the presence of Alzheimer's pathology (Dubois et al., 2007, 2010; Sperling et al., 2011; Jack and Holtzman, 2013). The most validated $\mathrm{AD}$ biomarkers are cerebrospinal fluid (CSF) amyloid- $\beta_{1-42}$, total tau and phosphorylated tau as pathophysiological biomarkers of Alzheimer's pathology, and brain regional structural and metabolic changes, as topographical biomarkers (Dubois et al., 2007; Jack and Holtzman, 2013). However, it has been recently recognized that the topographical markers lack pathological specificity necessary for diagnosis, and that they are more useful for measuring the disease progression (Dubois et al., 2014). According to this view, in vivo evidence of Alzheimer's pathology, and thus preclinical AD stage, would constitute increased cerebral amyloid burden/decreased CSF amyloid level together with increased levels of total tau and phospho-tau. The general idea is that if a person with Alzheimer's pathology lives long enough, they will eventually progress to AD dementia. Thus, biomarkers-based diagnosis provides an opportunity to introduce early treatments that will attempt to delay appearance of dementia symptoms and disease development.

While the value of early diagnosis is beyond any dispute, the question of how to disentangle typical aging and preclinical $\mathrm{AD}$ remains open. This issue is further complicated by the heterogeneous nature of the preclinical AD. According to one model, preclinical AD comprises three stages: at stage 1 , only brain amyloidosis is evident; at stage 2, both cerebral amyloidosis and neurodegeneration are present; and at stage 3 , these features are further combined with subtle cognitive changes (Sperling et al., 2011). Two additional stages have been added to this model: stage 0 , which represents cognitively intact people without brain amyloidosis, neurodegeneration, or subtle cognitive changes, and suspected non-amyloid pathology (SNAP), which is characterized by normal amyloid markers and abnormal neurodegeneration markers and which is not necessarily related to AD (Jack et al.,

2012). While it is clear that preclinical AD is characterized by the absence of cognitive impairment

Received: 03 May 2015 Accepted: 10 August 2015 Published: 26 August 2015

Citation:

Kljajevic V (2015) Overestimating the effects of healthy aging. Front. Aging Neurosci. 7:164. doi: 10.3389/fnagi.2015.00164 
on objective measures of cognition, subtle cognitive changes that have been associated with this stage of disease remain poorly understood (Dubois et al., 2014).

Another model of preclinical AD has recently been proposed in the context of a cross-sectional study that assessed a populationbased sample of $985 \mathrm{CN}$ people aged 50-89. The model combines only amyloidosis [positive $\left(\mathrm{A}^{+}\right)$or negative $\left(\mathrm{A}^{-}\right)$] and neurodegeneration [positive $\left(\mathrm{N}^{+}\right)$or negative $\left(\mathrm{N}^{-}\right)$] status, leaving out subtle cognitive changes. The study revealed that the estimated population frequency of the $\mathrm{A}^{-} \mathrm{N}^{-}$sequence was $100 \%$ at age 50 , but only $17 \%$ at age 89 , with the $\mathrm{A}^{+} \mathrm{N}^{+}$sequence reaching $42 \%$ by age of 89 , whereas the frequency of the sequence $\mathrm{A}^{-} \mathrm{N}^{+}$was $24 \%$ by that age (Jack et al., 2014). Examples of pathological sequences leading to $\mathrm{AD}$ dementia are as follows: $\mathrm{A}^{-} \mathrm{N}^{-}$to $\mathrm{A}^{+} \mathrm{N}^{-}$to $\mathrm{A}^{+} \mathrm{N}^{+}$, and $\mathrm{A}^{-} \mathrm{N}^{-}$to $\mathrm{A}^{-} \mathrm{N}^{+}$to $\mathrm{A}^{+} \mathrm{N}^{+}$, whereas the two component sequence $\mathrm{A}^{-} \mathrm{N}^{-}$to $\mathrm{A}^{-} \mathrm{N}^{+}$is linked to heterogeneous underlying pathology (Jack et al., 2014). However, the neurodegenerative status in this study was determined based on topographical biomarkers of $\mathrm{AD}$, more specifically by an $\mathrm{AD}$ signature ${ }^{18}$ F-fluorodeoxyglucose $\left({ }^{18} \mathrm{~F}\right.$-FDG $)$ PET and hippocampal volume on MRI. These topographical markers are not unique markers of Alzheimer's pathology; for example, hippocampal volume is reduced in other conditions, such as frontotemporal dementia, hippocampal sclerosis, Lewy-related pathology, argyrophilic grain disease, diabetes, and bipolar disorder, among others (Jack et al., 2012; Dubois et al., 2014). Thus, although these findings still suggest that pathological aging is a predominant way of cognitive aging, they also indicate a need for consensus on which biomarkers are suggestive of Alzheimer's pathology only and thus better suited for untangling preclinical $\mathrm{AD}$ and the healthy brain aging.

As models of preclinical $\mathrm{AD}$ continue to develop, a challenge to the field is to reconcile the evidence of $\mathrm{AD}$-related pathology found in a large number of CN elderly people (Jack et al., 2012, 2014) with the notion of "healthy" or "successful" aging (Rowe and Kahn, 1987). This evidence seems to question the research practice of not considering possible presence of Alzheimer's pathology in $\mathrm{CN}$ elderly participants when including healthy elderly persons in cognitive studies. However, without the actual evidence to exclude Alzheimer's pathology, one can assume that some

\section{References}

Bateman, R. (2015). Alzheimer's disease and other dementias: advances in 2014. Lancet Neurol. 14, 4-6. doi:10.1016/S1474-4422(14)70301-1

Brier, M. R., Thomas, J. B., Snyder, A. Z., Wang, L., Fagan, A. M., Benzinger, T., et al. (2014). Unrecognized preclinical Alzheimer disease confounds rs-fcMRI studies of normal aging. Neurology 83, 1-7. doi:10.1212/WNL. 0000000000000939

Dubois, B., Feldman, H. H., Jacova, C., Cummings, J. L., Dekosky, S. T., BarbergerGateau, P., et al. (2010). Revising the definition of Alzheimer's disease: a new lexicon. Lancet Neurol. 9, 1118-1127. doi:10.1016/S1474-4422(10)70223-4

Dubois, B., Feldman, H. H., Jacova, C., Dekosky, S. T., Barberger-Gateau, P., Cummings, J., et al. (2007). Research criteria for the diagnosis of Alzheimer's disease: revising the NINCDS-ADRDA criteria. Lancet Neurol. 6, 734-746. doi: 10.1016/S1474-4422(07)70178-3

Dubois, B., Feldman, H. H., Jacova, C., Hampel, H., Molinuevo, J. L., Blennow, K., et al. (2014). Advancing research diagnostic criteria for Alzheimer's disease: the IWG2 criteria. Lancet Neurol. 13, 614-629. doi:10.1016/S1474-4422(14)70090-0 percentage of $\mathrm{CN}$ elderly subjects in such studies may represent preclinical AD. This problem has been occasionally recognized (Gold et al., 2013; Brier et al., 2014). It clearly requires a systematic change in approach, because subtle cognitive changes, reliance on cognitive strategies, and networks' reorganization that one would interpret as the effects of healthy aging might actually reflect the disease progression. While the number of studies investigating that the impact of atrophy, hypometabolism, white matter changes, and ApoE4 on cognitive processes across the AD stages is consistently growing, possible effects of $\beta$-amyloid, t-tau, and p-tau on cognitive processes in preclinical $A D$ remain largely unexplored (Riedel, 2014).

Finally, in addition to apparently small percentage of $\mathrm{CN}$ persons who despite an advanced age resist Alzheimer's pathology (Jack et al., 2012, 2014), there exist so-called SuperAgers. These are elderly people $(80+)$ who appear to have healthy brains and well-preserved memory abilities. A recent study involving 12 SuperAgers found that their memory abilities were comparable to those of a group of healthy 50- and 65-year-old persons. When compared to a group of healthy age-matched peers on measures of cortical thickness, the SuperAgers had significantly thicker cerebral cortex. Furthermore, the left anterior cingulate was significantly thicker in SuperAgers compared to both groups (Harrison et al., 2012). Thus, we find not only neuropathology but also healthy brains and preserved memory at well-advanced age.

In conclusion, it is now possible to establish the presence/absence of Alzheimer's pathology in vivo by measuring parameters that indicate biological changes caused by $\mathrm{AD}$, thereby determining if a person is at risk for developing $\mathrm{AD}$. Incorporating such evidence into cognitive aging research allows a differentiation of possible influences of Alzheimer's pathology from the effects of healthy aging on cognitive processes. Only by systematically incorporating such evidence in research on cognitive aging, we will be able to make a progress in disentangling preclinical AD from healthy cognitive aging.

\section{Acknowledgments}

The author gratefully acknowledges support from IKERBASQUE, Basque Foundation for Science (111407EMDD).

Gold, B. T., Johnson, N. F., and Powell, D. K. (2013). Lifelong bilingualism contributes to cognitive reserve against white matter integrity declines in aging. Neuropsychologia 51, 2841-2846. doi:10.1016/j.neuropsychologia.2013. 09.037

Harrison, T. M., Weintraub, S., Mesulam, M. M., and Rogalski, E. (2012). Superior memory and higher cortical volumes in unusually successful cognitive aging. J. Int. Neuropsychol. Soc. 18, 1081-1085. doi:10.1016/j.neuropsychologia. 2013.09.037

Jack, C. R. Jr, and Holtzman, D. M. (2013). Biomarker modeling of Alzheimer's disease. Neuron 80, 1347-1358. doi:10.1016/j.neuron.2013.12.003

Jack, C. R. Jr, Knopman, D. S., Weigand, S. D., Wiste, H. J., Vemuri, P., Lowe, V., et al. (2012). An operational approach to NIA-AA criteria for preclinical Alzheimer's disease. Ann. Neurol. 71, 765-775. doi:10.1002/ana.22628

Jack, C. R. Jr, Wiste, H. J., Weigand, S. D., Rocca, W. A., Knopman, D. S., Mielke, M. M., et al. (2014). Age-specific population frequencies of cerebral $\beta$-amyloidosis and neurodegeneration among people with normal cognitive function aged 50 89 years: a cross-sectional study. Lancet Neurol. 13, 997-1005. doi:10.1016/ S1474-4422(14)70194-2 
Riedel, W. J. (2014). Preventing cognitive decline in preclinical Alzheimer's disease. Curr. Opin. Pharmacol. 14, 18-22. doi:10.1016/j.coph.2013.10.002

Rowe, J. H., and Kahn, R. L. (1987). Human aging: usual and successful. Science 237, 143-149. doi:10.1126/science.3299702

Selkoe, D. J. (2012). Preventing Alzheimer's disease. Science 337, 1488-1492. doi:10. 1126/science. 1228541

Sperling, R. A., Aisen, P. S., Beckett, L. A., Bennett, D. A., Craft, S., Fagan, A. M., et al. (2011). Toward defining the preclinical stages of Alzheimer's disease: recommendations from the National Institute on Aging-Alzheimer's association workgroups on diagnostic guidelines for Alzheimer's disease. Alzheimers Dement. 7, 280-292. doi:10.1016/j.jalz.2011.03.003
Conflict of Interest Statement: The author declares that the research was conducted in the absence of any commercial or financial relationships that could be construed as a potential conflict of interest.

Copyright (C) 2015 Kljajevic. This is an open-access article distributed under the terms of the Creative Commons Attribution License (CC BY). The use, distribution or reproduction in other forums is permitted, provided the original author(s) or licensor are credited and that the original publication in this journal is cited, in accordance with accepted academic practice. No use, distribution or reproduction is permitted which does not comply with these terms. 